\title{
MRI-Guided Biopsy as a Tool for Diagnosis and Research of Muscle Disorders
}

\author{
Saskia Lassche ${ }^{\mathrm{a}, *}$, Barbara H. Janssen ${ }^{\mathrm{b}}$, Ties IJzermans ${ }^{\mathrm{a}}$, Jurgen J. Fütterer ${ }^{\mathrm{b}}$, Nicol C. Voermans ${ }^{\mathrm{a}}$, \\ Arend Heerschap ${ }^{\mathrm{b}}$, Benno Küsters ${ }^{\mathrm{c}}$, Ritse M. Mann ${ }^{\mathrm{b}}$ and Baziel GM van Engelen ${ }^{\mathrm{a}}$ \\ ${ }^{a}$ Department of Neurology, Donders Institute for Brain, Cognition and Behaviour, Radboud University Medical \\ Center, The Netherlands \\ ${ }^{\mathrm{b}}$ Department of Radiology and Nuclear Medicine, Radboud University Medical Center, The Netherlands \\ ${ }^{\mathrm{c}}$ Department of Pathology, Radboud University Medical Center, The Netherlands
}

\begin{abstract}
.
Background: Sampling error is a common problem in muscle biopsies. MRI-guided biopsy allows verification of biopsy site during the procedure, which may reduce sampling error in patients with focal disease.

Objective: To describe the technique for MRI-guided muscle biopsy and discuss potential applications.

Methods: Axial MRI images were acquired to determine the target site for muscle biopsy. Needle trajectory was planned on 3D T1 weighted imaging and a MRI-guided biopsy of the vastus lateralis was performed in 13 FSHD patients.

Results: An adequate amount of muscle tissue was obtained in all participants, and MRI-guided biopsy succeeded in reaching focal target sites. However, symptomatic hematomas were observed in $2 / 13$ patients

Discussion: MRI-guided biopsy has a higher complication rate compared to traditional needle biopsy, most likely due to proximity to blood vessels in combination with the vacuum-assisted suction of the MRI-guided technique. We recommend that this technique is reserved for select diagnostic cases and research questions, with careful assessment of vasculature and reduced suction levels.
\end{abstract}

Keywords: Muscle MRI, muscle biopsy, facioscapulohumoral muscular dystrophy, inflammatory myopathy, myositis

\section{INTRODUCTION}

Histopathological analysis of muscle biopsies is a cornerstone of the diagnosis of muscle disorders. However, sampling error is a common problem. In inflammatory myopathies, false-negative results occur in 10-20\% of all biopsies due to focal disease activity $[1,2]$. In acquired and hereditary muscle disorders, fatty infiltration - a marker of disease severity - can vary from mild to severe within a single muscle $[3,4]$. Sampling of very early or end-stage muscle

\footnotetext{
${ }^{*}$ Correspondence to: Saskia Lassche, MD, Radboud university Medical Center, Department of Neurology, Postbus 9101, 6500 HB Nijmegen, The Netherlands. Tel.: +31 2436 16600; E-mail: Saskia.Lassche@radboudumc.nl.
}

may result in the absence of disease-specific abnormalities, impeding correct diagnosis.

In neuromuscular research, tissue-based studies often employ biopsy samples from muscles with variable disease severity. Correlation with muscle imaging may reduce variability and enhance studies that rely on an association with disease severity.

Magnetic resonance imaging (MRI) allows accurate identification of areas of inflammation and fatty infiltration in hereditary and inflammatory myopathies. MRI triage has been used to reduce sampling error in the diagnosis of inflammatory myopathies by selecting affected muscles prior to muscle biopsy [5, 6]. However, biopsies obtained from muscles with inflammation on MRI still had a 
false-negative rate of 0.19 [5]. This suggests that more precise targeting may improve diagnostic yield, in particular in patients with small, focal abnormalities.

MRI-guided biopsy using MRI-compatible needles allows verification of biopsy site during the procedure and is an established technique in the diagnosis of prostate and breast cancer [7, 8]. Here we demonstrate that MRI-guided biopsy can be used to acquire muscle biopsies in patients with muscle disorders and discuss potential applications.

\section{MATERIALS AND METHODS}

\section{Patients and study design}

We performed MRI-guided muscle biopsy in 13 genetically confirmed FSHD patients that were undergoing muscle biopsy of the vastus lateralis as part of a separate study [9]. Ethical approval was obtained from the regional medical ethics review committee and written informed consent received from all participants.

\section{Muscle imaging and MRI-guided biopsy procedure}

\section{Muscle imaging}

Axial T1 weighted and Turbo Inversion Recovery Magnitude (TIRM) images were obtained to determine the appropriate target area for muscle biopsy. MR measurements were conducted using a 3 Tesla MR system (Tim TRIO, Siemens, Erlangen, Germany) with patients being scanned in supine position. A fish oil capsule was placed at one third of the distance between the anterior superior iliac spine and the patella as an external reference point. To obtain maximum quality images, we used a spine array coil and two phased array coils covering the patient's legs. For thigh muscle imaging, the table was positioned to ensure that the upper leg was in the isocenter of the magnetic field. Scout images were acquired in three orthogonal directions for accurate positioning of the MRI slices. Thereafter 8 transversal slices (FOV $175 \times 175 \mathrm{~mm}^{2}$, thickness $4 \mathrm{~mm}$, gap $6 \mathrm{~mm}$, base resolution 256) were acquired with a T2 multi spin echo sequence (TR: $3000 \mathrm{~ms}, 16$ equally spaced echo times $7.7-123.2 \mathrm{~ms}$ ). Subsequently followed by 23 transversal slices (thickness $4 \mathrm{~mm}$, gap $0.4 \mathrm{~mm}$ ) obtained with a T1 turbo spin echo sequence (FOV $250 \times 244.5 \mathrm{~mm}^{2}$, TR/TE $600 \mathrm{~ms} / 13 \mathrm{~ms}$, baseresolution 448), and with a Turbo Inversion Recovery sequence $\left(\right.$ TIRM) $\left(\right.$ FOV $175 \times 175 \mathrm{~mm}^{2}$, TR/TE/TI
$4100 \mathrm{~ms} / 41 \mathrm{~ms} / 220 \mathrm{~ms}$, baseresolution 256). As a rule, we scanned the right leg unless manual muscle testing demonstrated asymmetrical weakness; in these cases the most severely affected leg was examined and biopsied.

Target area and needle trajectory The appropriate target area for the MRI-guided biopsy was determined on axial images. Choice of target area was informed by the amount and location of remaining muscle tissue, fatty infiltration, and the presence of hyperintensities on TIRM suggestive of inflammation. In radiologically normal muscle tissue, the target area was set in the center of the vastus lateralis at the level of the fish oil marker (Fig. 1). The trajectory of the biopsy needle (i.e., incision site, angle, and depth) was constructed based on anatomical 3D T1-weighted imaging (Fig. 1; field of view $269 \times 269 \times 160 \mathrm{~mm}$; base resolution 384 ; repetition time $735 \mathrm{~ms}$; echo time 2,54 ms).

\section{MRI-guided biopsy}

After the needle trajectory had been determined, and with the patient still positioned on the MRI table, local anesthesia (lidocaine 2\%) was injected subcutaneously. Taking routine antiseptic precautions, a 4-mm incision was made and an MR-compatible biopsy trocar inserted (Fig. 2). A repeat T1weighted 3D scan was performed to verify the position of the needle. If necessary, the trocar was repositioned accordingly. An experienced interventional radiologist performed the biopsy using a 9-gauge $(3.7 \mathrm{~mm})$ vacuum-assisted biopsy needle (Suros ATEC, Hologic, Bedford, USA). Subsequently, another 3D T1-weighted image as well as axial TIRM images were obtained to confirm the final biopsy site and to assess complications. The incision site was closed using steri-strips, after which a pressure bandage was applied. Positioning of the patient and diagnostic imaging took approximately 20 minutes. The MRI-guided biopsy procedure took an additional 20-50 minutes depending on the complexity of the target site.

\section{Histology}

Biopsy material was frozen in isopentane and stored at $-80^{\circ} \mathrm{C}$. Biopsy sections were stained with $\mathrm{H}$-Phlox to assess fiber size variability, presence of internal nuclei, the destruction of muscle fibers and regeneration, as well as supporting connective tissue. 

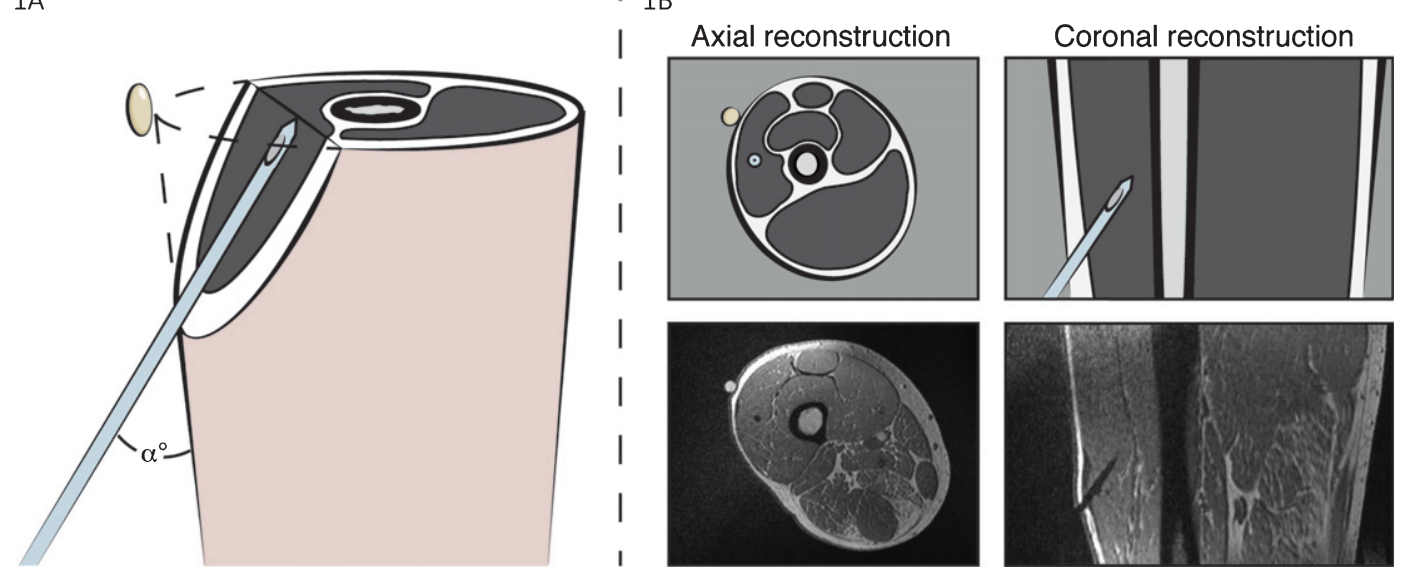

Fig. 1. MRI-guided biopsy of the right vastus lateralis. (A) The exact needle trajectory to the target area was determined by manually shifting anatomical 3D T1 images in at least three orthogonal planes. The appropriate needle angle $\left(\alpha^{\circ}\right)$, depth, and insertion site relative to the fish oil marker was based on these images. (B) A needle trocar was inserted after which a subsequent 3D T1 was obtained to verify the position of the needle tip in the $\mathrm{m}$. vastus lateralis. If necessary, the trocar was adjusted and repeat verification imaging performed. After positioning the needle into the correct site, a vacuum-assisted biopsy was taken.

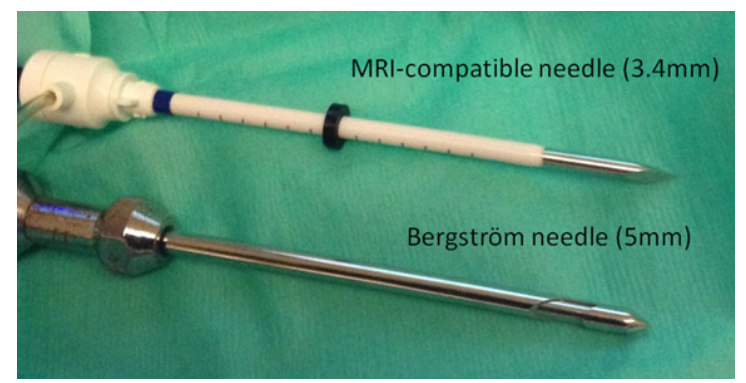

Fig. 2. MRI-guided biopsy needle. Comparison between $3.4 \mathrm{~mm}$ MRI-compatible needle for MRI-guided biopsy and $5 \mathrm{~mm}$ routine Bergström needle.

\section{RESULTS}

\section{MRI-guided biopsy procedures}

MRI images showed pathological changes compatible with FSHD [3]. No or very minor fatty infiltration was observed in $8 / 13$ participants. In these participants a biopsy was taken in the center of the vastus lateralis, at or slightly above or below the reference marker. In the $5 / 13$ participants with fatty infiltration we were able to obtain a biopsy from the intended target site. All biopsies from muscles with moderateto-severe fatty infiltration ( $>30 \%$ ) showed dystrophic changes, whereas all biopsies from muscles with normal or mild fatty infiltration $(<30 \%)$ were normal or showed mild myopathic abnormalities.

Symptomatic post-biopsy hematomas, resulting in self-limiting local pain and swelling, were observed in $2 / 13$ patients and asymptomatic hematomas were observed on control imaging in another 1/13. In all 3 patients a blood vessel could be identified close to the biopsy site. Because bleeding complications are very rare in routine needle biopsies, it seems likely that the stronger suction used with MRI-guided biopsy contributed to hematoma formation.

Tissue size was similar or larger than routine needle biopsies, yielding an adequate amount of tissue to perform histological evaluation in all, and enough tissue for extensive additional research evaluations in 12/13 participants.

Participants reported a pain score of $5.2 \pm 0.7$ out of 10 after the MRI-guided muscle biopsy. Apart from pain associated with the biopsy, they also complained about discomfort due to lying on the MRI table for an extended period of time.

\section{Case study - MRI-guided biopsy in a patient with limited residual muscle tissue}

In a participant with severe FSHD (Clinical Severity Score 8/10) we observed extensive fatty infiltration of all upper leg muscles (Fig. 3A). Routine needle biopsy without imaging would yield only fat. Using MRI-guided biopsy we were able to position the needle within the area with residual muscle tissue and obtain a tissue specimen for analysis (Fig. 3B-F). Muscle biopsies from severely affected muscle as in this example may inform research studies, but have limited relevance for diagnostic studies, as diseasespecific abnormalities may be absent in end-stage 

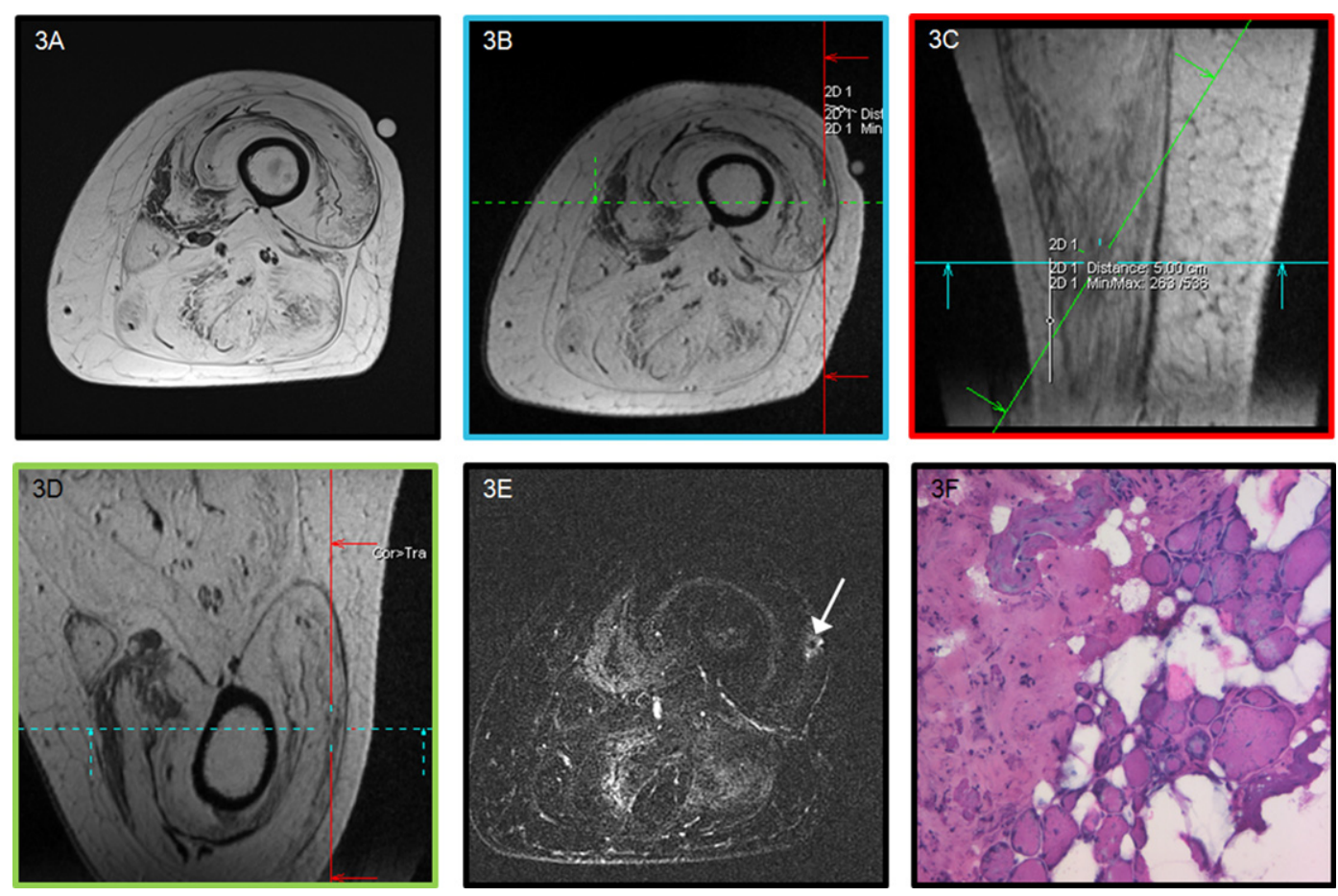

Fig. 3. MRI-guided muscle biopsy in a patient with limited residual muscle tissue. (A) Axial T1-weighted axial image of the left upper leg of a 49-year-old female FSHD1 patient showing marked fatty infiltration of all muscles. (B-D) 3D T1 planning images to determine the appropriate needle trajectory to the target site. Colored boxes surrounding the images correspond with colored lines indicating images planes. (E) post-biopsy TIRM image showing the biopsy site (arrow). (F) H-Phlox staining demonstrates severe dystrophic changes as reflected by the marked increase in fiber size variability, increased internal nuclei, fibrosis, and fatty infiltration.

muscle. However, this case study demonstrates that the MRI-guided technique is capable of targeting very specific target areas, which could also be applied to focal areas of inflammation.

\section{DISCUSSION}

This study demonstrates that MRI-guided biopsy can be used in patients with muscle disease to obtain muscle tissue from specific target areas. MRI-guided biopsy enables verification of the muscle biopsy site during the procedure, which allows selective targeting and definite confirmation of the biopsy site on MRI, as well as a direct assessment of potential complications.

MRI-guided biopsy enabled us to acquire tissue in an individual with extensive fatty infiltration, which demonstrates the ability of this technique to target focal areas of residual muscle tissue or localized inflammation. This may benefit diagnostic testing; e.g. to discriminate neurogenic from myopathic causes in individuals with severe disease; or to target focal areas of inflammation to confirm the presence of myositis prior to treatment with immunosuppressant therapy.

Aside from potential diagnostic application, MRIguided biopsy could also a valuable tool in research studies where precise radiological - tissue correlation is required. Furthermore, the addition of MRI makes it possible to revisit a specific site for repeat biopsy in clinical trials that use longitudinal muscle biopsies to assess therapeutic efficacy.

Although we did successfully obtain muscle tissue from the intended biopsy site in all participants, this resulted in symptomatic hematomas in $2 / 13$ patients and an asymptomatic hematoma in another. Complications following routine Bergström needle biopsy are rare. In a retrospective analysis of 13,636 Bergström needle biopsies, hematomas or arterial bleeding were observed in 3 and perceptible and bothersome pain $>3$ days were observed in 5 procedures [10]. The higher rate of hematomas in our study can be attributed to biopsy position within the muscle in combination with strong vacuum suction applied during the MRI-guided biopsy. Additionally, postbiopsy imaging will identify hematomas that would 
otherwise have gone unnoticed, forewarning the clinician and patient of potential pain and swelling.

The amount of pain reported by patients is similar to pain scores after routine Bergström needle biopsy in our center $(5.2 \pm 0.7$ vs. $6.0 \pm 2.1)$.

This study demonstrates that MRI-guided biopsy of focal abnormalities or in individuals with extensive fatty infiltration is feasible in patients with muscle disorders. However, we observed a higher rate of symptomatic bleeding complications compared to routine Bergström needle biopsy. In addition to this, MRI-guided biopsy is more expensive than routine needle biopsy due to the use of MRI, longer procedure time and use of disposable materials. As this proof-of-principle study addresses the feasibility of the technique we performed biopsies in a homogenous group of patients with the same disease and in the same muscle. However, the same methodology can be applied to target different muscles or different diseases. Histopathological analysis matched the severity of fatty infiltration, however future studies may address the diagnostic value of MRI-guided biopsy and the correlation between imaging signal and histopathology more precisely.

A potential diagnostic application of MRI-guided biopsy is targeting of focal inflammation that potentially requires treatment with immunosuppressants, where a diagnosis cannot be made with Bergström needle biopsy of MRI-positive muscles. Another potential diagnostic application is to confirm the presence of a myopathic process in patients with severe disease. In addition to diagnostic testing, MRI-guided biopsy may have potential for research where targeting of a specific muscle site is required. In these case, careful assessment of vasculature and reduced vacuum-assisted suction may reduce bleeding complications.

\section{AUTHOR CONTRIBUTIONS}

SL conceived the study, was responsible for data collection and analysis, and drafted the manuscript. BHJ designed the MRI protocol, was involved in data collection and analysis, and drafted the manuscript. TI contributed to the data collection and analysis, and drafted the manuscript. JJF conceived the study, performed biopsies, and was involved in the revision of the manuscript. NCV assisted in data collection and revised the manuscript. BK performed the histological evaluations and revised the manuscript. $\mathrm{AH}$ supervised BHJ and revised the manuscript. RM performed biopsies and revised the manuscript. BGMvE conceived the study and revised the manuscript. All authors have read and approved the final article.

\section{ACKNOWLEDGMENTS}

This study is supported by the Prinses Beatrix Spierfonds and Stichting Spieren voor Spieren (W.OR10-30). B. Janssen is supported by the Prinses Beatrix Spierfonds (WAR08-15) and the FSHD Stichting (WP15).

\section{CONFLICTS OF INTEREST}

The authors have no conflicts of interest to report.

\section{REFERENCES}

[1] Bohan A, Peter JB, Bowman RL, Pearson CM. Computerassisted analysis of 153 patients with polymyositis and dermatomyositis. Medicine (Baltimore). 1977;56(4):25586.

[2] Amato AA, Barohn RJ. Evaluation and treatment of inflammatory myopathies. J Neurol Neurosurg Psychiatry. 2009;80(10):1060-8.

[3] Janssen BH, Voet NB, Nabuurs CI, Kan HE, de Rooy JW, Geurts AC, et al. Distinct disease phases in muscles of facioscapulohumeral dystrophy patients identified by MR detected fat infiltration. PloS One. 2014;9(1):e85416.

[4] Tasca G, Pescatori M, Monforte M, Mirabella M, Iannaccone E, Frusciante R, et al. Different molecular signatures in magnetic resonance imaging-staged facioscapulohumeral muscular dystrophy muscles. PloS One. 2012;7(6): e38779.

[5] van de Vlekkert J, Maas M, Hoogendijk JE, de Visser M, van Schaik IN. Combining MRI and muscle biopsy improves diagnostic accuracy in subacute-onset idiopathic inflammatory myopathy. Muscle Nerve. 2015;51(2):253-8.

[6] Schweitzer ME, Fort J. Cost-effectiveness of MR imaging in evaluating polymyositis. AJR Am J Roentgenol. 1995;165(6):1469-71.

[7] Moore CM, Robertson NL, Arsanious N, Middleton T, Villers A, Klotz L, et al. Image-guided prostate biopsy using magnetic resonance imaging-derived targets: A systematic review. European Urology. 2013;63(1):125-40.

[8] Price ER. Magnetic resonance imaging-guided biopsy of the breast: Fundamentals and finer points. Magnetic Resonance Imaging Clinics of North America. 2013;21(3):571-81.

[9] Lassche S, Ottenheijm CA, Voermans NC, Westeneng HJ, Janssen BH, van der Maarel SM, et al. Determining the role of sarcomeric proteins in facioscapulohumeral muscular dystrophy: A study protocol. BMC Neurology. 2013;13:144.

[10] Tarnopolsky MA, Pearce E, Smith K, Lach B. Suctionmodified Bergstrom muscle biopsy technique: Experience with 13,500 procedures. Muscle Nerve. 2011;43(5):717-25. 